\title{
When do performance-based risk-sharing arrangements make sense?
}

\author{
Michael Drummond ${ }^{1}$
}

Published online: 18 March 2015

(c) Springer-Verlag Berlin Heidelberg 2015

\section{Introduction}

Performance-based risk-sharing arrangements (PBRSAs) are schemes in which the performance of a new health technology is tracked in a defined patient population over a period of time and the amount or level of reimbursement is based on the cost and health outcomes achieved [1-4]. These schemes go under different names in different jurisdictions (e.g. "risk-sharing", "coverage with evidence development", “only with research", "field evaluations"), but have the common feature that the technology concerned is granted reimbursement on the condition that more data are collected and then a final price and/or reimbursement status for the technology determined based on the results of the data collection exercise.

In principle, these schemes are attractive to both technology manufacturers, since products may gain market access that otherwise might be rejected, and the payers for health care, since the risk of paying for technologies that are a poor value for the money is minimized. However, experience with such schemes has been mixed. For example, in the United Kingdom, the scheme for bortezomib, a drug for multiple myeloma, is widely regarded as a "success", but the scheme for multiple sclerosis drugs is widely regarded as a "failure" [5]. Therefore, if such schemes are to be used more widely in the future, it is important to be absolutely clear on the circumstances where they make sense and where they do not. Therefore, those embarking on such schemes pose the following questions.

Michael Drummond

mike.drummond@york.ac.uk

1 Centre for Health Economics, Alcuin A Block, University of York, Heslington, York YO10 5DD, UK
Is there uncertainty about the clinical or economic benefits of the technology that can be reduced by further study?

Trends in the licensing of drugs (e.g., "fast track" approval) mean that new products can obtain approval to market with evidence that, for various reasons, payers may not find sufficiently comprehensive. Other technologies, such as medical devices and surgical procedures, may not even have to generate evidence of clinical effectiveness from randomized controlled trials in order to be marketed. So uncertainty about clinical and economic outcomes is a prime motivation for PBRSAs. However, some types of uncertainty (e.g., over whether a previously unrecognized adverse effect may emerge in the long term, or whether changes in market conditions might cause the price of the technology to drop in the future) cannot be easily resolved by further study. Therefore, one should not embark on PBRSAs to postpone a reimbursement decision that should be taken today, albeit with the possibility of revision at a later date. This might be one of the explanations for the shift, in the United Kingdom, from PBRSAs to "patient access schemes" that involve price cuts, as opposed to requests for further research.

\section{Can the relevant clinical or economic outcomes be clearly defined and measured in a satisfactory manner?}

Prior to embarking on a PBRSA, it is useful to have access to a decision-analytic model that facilitates exploration of the relationships between the key parameters that affect costs and benefits in order to estimate the values that they 
would have to achieve in order for the technology to be cost-effective. Typically, the main interest in PBRSAs is in the relative clinical effectiveness of the new technology, as compared with the current standard of care, particularly in the long term, or in particular sub-groups of the patient population. Occasionally, the outcome of interest may be relatively short term, such as M-protein as a measure of response in the bortezomib example given above, but normally longer-term follow-up is required.

Therefore, PBRSAs are often considered in situations where approval to market was granted based on improvements in an intermediate, or surrogate, outcome, and where further study may help determine whether improvements in length and/or quality of life are delivered as a result. For example, in the field of cancer therapy, clinical trials are often terminated when it is shown that the new therapy is superior in delaying disease progression, but there is mixed evidence on the reliability of progression-free survival as a surrogate for predicting overall survival [6]. Since it is extremely difficult, although not impossible, to secure agreement to a randomized study once a technology is reimbursed, it is worth considering whether convincing evidence on effectiveness can be gathered in an observational study.

It is also important to be sure that the outcome of interest is largely influenced by the technology concerned. One of the first-ever PBRSAs concerned a drug for benign prostatic hyperplasia in Canada. The basis of the scheme was that if patients given the drug required surgery after 1 full year of medical therapy, the costs of the drug therapy would be refunded [2]. However, this assumes that the decision as to whether to operate depends entirely on the patient's health condition and not the aspirations of surgeons!

\section{Are the timelines for the scheme reasonable?}

Two timelines are important: the time required to initiate the scheme and the time required to conduct the necessary research. Several attempted schemes have failed because of lack of agreement between the relevant parties. Depending on the technology being studied, these parties could include the payer (or organization proposing the scheme), the manufacturer, clinical associations (who may need to convince their members to participate), hospitals, and individual health care professionals (who may need to collect the data). The time required to negotiate a given scheme will depend on how contentious it is, but it would be wise to anticipate these discussions. Also, if individual PBSRAs are part of a broader programme or policy, it would be wise to obtain an outline agreement of all the relevant parties to collaborate in the programme.
Once the scheme is underway, the time required for the research will depend on the nature of the outcomes being studied and the research design. For example, it would normally take longer to recruit patients into an RCT than an observational study, particularly if the observational study were just relying on routinely available data. Those planning PBRSAs should be wary of schemes that require a follow-up longer than around 3 years. This is because the kinds of policy questions that such studies inform have the habit of changing, for example as other health technologies become available for the same patient group. One of the reasons for the "failure" of the PBRSA of multiple sclerosis drugs in the UK was that the results took a long time to be delivered and the government changed in the interim.

However, there are now several new technologies that will take several years for us to understand their full benefit. For example, some new drugs, for conditions such as CML and melanoma, offer the potential for a "cure" in that tumor suppression may be achievable in the long term. Also, gene therapy may become available, where a single administration could deliver a long-term benefit for the patient. Of course, we will not know how long clinical benefits are maintained until some patients have been on the therapy for a considerable length of time. This presents a major challenge to payers in rewarding the value added by these therapies, since the cost is borne today but the potential benefits stretch far into the future. One proposed approach is to "lease" the technology for a defined period of time and to renew the lease as long as the technology is still delivering the desired benefit [7] Whether or not it is labeled as a PBRSA, such a scheme would clearly involve long-term data collection of the type discussed above.

\section{Will the arrangements for data collection and analysis be easily implementable and affordable?}

As in any research project, issues such as who will collect the data and who will analyze it need to be addressed. The method of their resolution will depend on the nature of the study and the setting(s) in which it takes place, but attention needs to be paid to maintaining patient confidentiality and the independence of the analysis. In addition, there is the question of who pays for the study. In jurisdictions where schemes can be proposed by manufacturers, the manufacturer is normally required to fund the research, although the technology itself is reimbursed. In a few jurisdictions where the schemes are proposed by the payer, there is sometimes a budget for undertaking the research, as in the case of the "field evaluations" undertaken in Ontario [8]. 
The simpler the scheme and the greater the reliance on routinely available data, the cheaper it is likely to be. There are differences of opinion about the level of complexity of PBRSAs. While simpler schemes are likely to be cheaper to implement, more complex schemes make it harder to calculate the level of any implied price discount. This has some attraction for manufacturers who may be concerned about international price referencing.

\section{Can clarity be reached on the likely decisions following the data collection and analysis?}

The purpose of PBRSAs is to help us reach the optimal pricing and reimbursement decision for a given technology. The first point to note is that "optimal" may be more or less easy to define from one country to another, depending on the clarity of the decision rule for adopting new technologies (for example, it might be clearest in a country employing an explicit cost-effectiveness threshold). Whatever the decision rule, it is preferable that specific targets are specified, linking specific outcomes from the research with particular pricing and reimbursement decisions.

In some jurisdictions, this has proved a challenge, mainly because payers find it hard to specify what the funding challenges are likely to be in (say) 3 years time, since these will depend on the extent of the budgetary constraint and the number of other new technologies becoming available over that time period. However, it is worthwhile trying to address these challenges, because uncertainty about the decision outcome is the most oftenquoted concern of manufacturers when being asked to participate in these schemes.

In conclusion, PBRSAs do represent a useful mechanism for all parties to work together to improve pricing and reimbursement decisions for new technologies in cases where there is uncertainty about future clinical and economic outcomes. However, this is a clear case of the devil being in the details. Attention to the points raised here may help those devising such schemes improve their chances of success.

\section{References}

1. Hutton, J., Trueman, P., Henshall, C.: Coverage with evidence development: an examination of conceptual and policy issues. Int $\mathrm{J}$ Tech Assess Health Care 23, 425-435 (2007)

2. Adamski, J., Godman, B., Ofierska-Sujkowska, G., et al.: Risk sharing arrangements for pharmaceuticals: potential considerations and recommendations for European payers. BMC Health Serv Res 10, 153-169 (2010)

3. Garrison, L.P., Towse, A., Briggs, A., et al.: Performance-based risk-sharing arrangements- Good practices for design, implementation and evaluation: report of the ISPOR good research practices for performance-based risk-sharing arrangements task force. Value Health 16, 703-719 (2013)

4. Neumann, P.J.: Where are we on 'risk-sharing' arrangements? Value Health 16, 701-702 (2013)

5. Raftery, J.: Multiple sclerosis risk sharing scheme: a costly failure. Br Med J (2010). doi:10.1136/bmj.c1786

6. Ciani, O., Davies, S., Tappenden, P., et al.: Validation of surrogate endpoints in advanced solid tumors: systematic review of statistical methods, results, and implications for policy makers. Int $\mathbf{J}$ Tech Assess Health Care 30, 312-324 (2014)

7. Edlin, R., Hall, P., Wallner, K., McCabe, C.: Sharing risk between payer and provider by leasing health technologies: an affordable and effective reimbursement strategy for innovative technologies? Value Health 17, 438-444 (2014)

8. Levin, L., Goeree, R., Sikich, N., et al.: Establishing a comprehensive continuum from an evidentiary base to policy development for health technologies: the Ontario experience. Int J Tech Assess Health Care 23(3), 299-309 (2007) 